\title{
Aviptadil: Class Effect of a Synthetic Vasoactive Intestinal Peptide as a Treatment Option in Patients with COVID-19 with Severe Respiratory Failure
}

\author{
Authors:

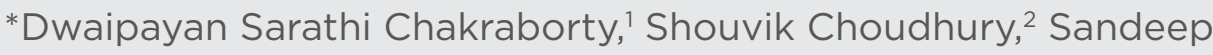 \\ Lahiry $^{3}$ \\ 1. Department of Pharmacology, Diamond Harbour Government Medical College and \\ Hospital, West Bengal, India \\ 2. Department of Pharmacology, Burdwan Medical College, West Bengal, India \\ 3. Kolkata, West Bengal, India \\ *Correspondence to drdsc2014@gmail.com
}

Disclosure: $\quad$ The authors have declared no conflicts of interest.

Received: $\quad 12.10 .21$

Accepted: $\quad 29.11 .21$

Keywords: Interim, pandemic, severe acute respiratory syndrome coronavirus 2 (SARS-CoV-2), synthetic, vasoactive intestinal peptide (VIP).

Citation: $\quad$ EMJ Microbiol Infect Dis. 2022; DOI/10.33590/emjmicrobiolinfectdis/21-00222.

\section{Abstract}

Despite dynamic drug and vaccine development processes to reduce the disease burden of COVID-19, the treatment options are still very limited. Vasoactive intestinal peptide (VIP) has a diversified physiological action with specific features of lung protection-related activities.

VIP inhibits severe acute respiratory syndrome coronavirus 2 (SARS-CoV-2) gene replication in human monocytes and the viral replication in Calu-3 cells, thus further reducing the generation of proinflammatory mediators. Aviptadil, a synthetic form of VIP, is the only pulmonary therapeutic agent to have been granted 'fast track' status by the U.S. Food and Drug Administration (FDA) and to be allowed into both Phase II and III clinical trials. Initial binding of Aviptadil with non-structural protein (nsp) 10 and nsp16, which may inhibit the 2'-O-methyltransferase activity of the SARS-CoV-2 nsp10 and nsp16 complex.

Aviptadil has already proved to be an effective option in the treatment of severe respiratory failures due to sepsis and other related lung injuries. Interim analysis results of this drug used in respiratory failure caused by SARS-CoV-2 has evolved a new hope in regard to safety and efficacy. The final results from a recently completed trial, as well as all currently ongoing trials, will clarify the class effect of this drug in the treatment of COVID-19 in future days.

\section{INTRODUCTION}

COVID-19 has created an unprecedented global situation over the last 18 months. As of $23^{\text {rd }}$ November 2021, there have been 257,469,528 confirmed cases of COVID-19 reported to the

World Health Organization (WHO), including $5,158,211$ deaths worldwide. This pandemic has led to extensive research to evaluate the safety and efficacy of several repurposed but also some new drugs. Numerous clinical trials are ongoing to establish the clinical benefits of these drugs. 
The mainstay of the treatment continues to be based on supportive care, with the possible use of pharmacological agents in patients with more severe illnesses. Antiviral agents such as remdesivir may help to shorten the duration of illness but may not be efficacious enough to provide the survival benefit in life-threatening situations. Hypoxic individuals, as well as those requiring supplemental oxygen and non-invasive or invasive ventilatory support, treated with low-dose steroids have shown improved survival outcomes, with robust data supporting the statistically significant clinical outcome parameters.

In an adjunct to that, parenteral as well as oral anticoagulants have shown promising results in regard to combatting fatal complications such as a pulmonary embolism in cases of moderate-tosevere illness, where individuals are hospitalised in dedicated isolation wards as well as intensive care settings. Convalescent plasma has not lived up to the promise it held as the recent randomised clinical trials failed to demonstrate any added benefit as far as the mortality and morbidities of the disease are concerned. Cytokine inhibitors such as tocilizumab and other immunomodulatory drugs need further evaluation among a larger participating population of clinical trials to establish their efficacy.'

After performing an extensive literature review using three databases (PubMed, Scopus, and Cochrane), it has been found that, in spite of dynamic drug and vaccine development processes to reduce the disease burden of COVID-19, the disease may not be eradicated due to the evolution of newer mutant strains of severe acute respiratory syndrome coronavirus 2 (SARS-CoV-2) and logistical challenges in the administration of vaccines globally. Due to these reasons, the cornerstone of such disease management remains dependent, to a certain extent, on novel drug discoveries and their accelerated regulatory approval to be used on basis of investigational therapeutic tools. ${ }^{2}$

Vasoactive intestinal peptide (VIP) was first isolated from a hog's intestine by Said and Mutt in $1970 .^{3}$ It is mainly located in the myenteric and sub-mucosal neurons as well as nerve terminals of the gastrointestinal tract and contains 28 residue amino acid peptides. Apart from the digestive system, it is widely distributed in both the peripheral and central nervous systems as well as the cardiovascular, respiratory, and reproductive systems. This gut peptide hormone belongs to the glucagon/secretin hormone superfamily and is produced by neuroendocrine cells, macrophages, and both B and T Iymphocytes. ${ }^{4,5}$

\section{PHYSIOLOGY OF VASOACTIVE INTESTINAL PEPTIDES}

VIP is highly expressed in the lung tissue (approximately 70\%) and nasal mucosa. ${ }^{6}$ It exerts its action in the lung tissue via two types of receptors acting as $\mathrm{G}$ protein-couple receptors; VIP receptor Types 1 and 2 (VPAC1 and 2); and receptors that are also activated by the pituitary adenylate cyclase activating polypeptide, which belongs to the same family as VIP. The VPAC1 receptor is predominantly located in lung tissue and T lymphocytes whereas VPAC2 is found on smooth muscle, mast cell, and basal part of lung mucosa. ${ }^{7}$ VIP binds to alveolar Type ॥ (AT-II) cells via the VPAC1 receptor. ${ }^{8}$ Despite comprising of only $5 \%$ of the total lung tissue, it plays an important role in surfactant production, which helps in the maintenance of alveolar Type I epithelial cells.

VIP augments surfactant production by upregulating the enzyme choline phosphate cytidylyl transferase, which induces the incorporation of methyl choline to phosphatidylcholine, a major component of pulmonary surfactant. ${ }^{9}$ Additionally, it induces the c-Fos protein expression in AT-II cells and upregulates surfactant protein A expression, both of which ultimately lead to surfactant production. $^{10}$ It also inhibits apoptosis by blocking the activities of caspase, granzyme $B$, and perforin.11,12 It exerts non-adrenergic, non-cholinergic bronchodilatation, which is 100 times more potent than isoproterenol and 50 times more potent vasodilatation in both systemic and pulmonary arteries than prostacyclin.13,14 Preclinical experiments in mouse models have demonstrated their role in reducing ischaemia-induced reperfusion injuries. ${ }^{15}$

Besides the impact on respiratory physiology, VIP also has several other significant actions such as:

> Positive chronotropic, ionotropic, and coronary vasodilatory actions 
> Secretion of water and electrolyte on gastrointestinal lumen, pancreatic juice, and bile

> Stimulation of pepsinogen secretion

> Regulation of Prolactin secretion and promoting vaginal lubrication

> Inhibiting T Iymphocyte proliferation ${ }^{16}$

> Promoting T-helper 2 lymphocytes differentiation against T-helper lymphocytes and regulatory $T$ cell induction ${ }^{16,17}$

> Downregulating several macrophagemediated inflammatory cytokines and proinflammatory receptors ${ }^{18}$

> Playing a role of an inhibitory neurotransmitter of the non-adrenergic, non-cholinergic autonomic nervous system ${ }^{19}$

> Inhibiting the synthesis as well as activation of nuclear factor k-light-chain-enhancer of activated $B$ cells, which block the process of TNF-a generation ${ }^{20}$

\section{RATIONALITY BEHIND OF USE OF VASOACTIVE INTESTINAL PEPTIDES IN COVID-19}

Acute respiratory failure is a major cause of death due to the SARS-CoV-2 infection. It is attributed to a cytokine storm, preceded by the invasion of the alveolar cell by the virus itself and then rupture of that pulmonary epithelial cell. This invasion occurs once the virus enters the AT-II cell by binding its spike protein to the surface of the angiotensin-converting enzyme 2 receptors located on AT-II cells. ${ }^{21}$ AT-II cells express VPAC1 receptors on its surfaces, which the VIP binds to and prevents the process of apoptosis in lung injury. ${ }^{22}$ VIP inhibits SARS-CoV-2 gene replication in human monocytes and viral replication in Calu-3 cells, thus further reducing the generation of proinflammatory mediators that play a significant role in tissue injury in the course of the COVID-19 disease. ${ }^{23}$

VIP has demonstrated its beneficial effects on lung injuries in several animal models (Table 1). Unlike the anti-IL-6 drugs, it preserves the surfactant production as well as protects the AT-II cells of the lung. 4,23,28 Other than the surfactant producing and anti-inflammatory activity, VIP has the property of potentially inhibiting the Fas ligand expression and thereby halting the progression of Fas ligand-mediated cell death., ${ }^{9,17}$ Acute lung injury caused by the COVID-19 is also contributed to by the degranulation of serine proteases granzymes and the formation of perforin protein, which induces the rapid death of the target cells. ${ }^{21} \mathrm{As}$ a proven inhibitor of granzyme and perforin, VIP plays an important role in the prevention of cell death in lung tissue. ${ }^{29}$

\section{AVIPTADIL USE IN CLINICAL PRACTICE}

Aviptadil is a synthetic form of VIP, which is also known as RLF-100. It has been designated as an orphan drug by the U.S. Food and Drug Administration (FDA) to treat respiratory airway diseases such as asthma, chronic obstructive airway disease, cystic fibrosis, pulmonary hypertension, adult respiratory distress syndrome (ARDS), lung fibrosis, and sarcoidosis as well as in non-respiratory conditions such as erectile dysfunction."

It is available both intravenously and as inhalational preparations. The half-life of the drug is 1-2 min and the apparent volume of distribution is $14 \mathrm{~mL} / \mathrm{kg}$. This drug is almost eliminated by the renal route, where $35 \%$ elimination occurs in the first 4 hours and 90\% occurs within 24 hours. It has no significant clinical drug-drug interactions and insufficient data is available on its use in pregnancy and lactation. Intravenous administration is associated with side effects like tachycardia, flushing, hypotension, diarrhoea, and alterations in ECG (bigeminy). ${ }^{30}$

Patients with pulmonary arterial hypertension were successfully treated with inhaled aviptadil, which caused a reduction in pulmonary artery pressure and improved in cardiac output and mixed venous oxygen concentration. ${ }^{31}$ In another open label Phase II clinical study, 20 patients with histologically proven sarcoidosis were treated with inhaled aviptadil for 4 weeks, which caused a significant reduction of TNF-a and the increment of CD4+ CD127- CD25+ T cells in their bronchoalveolar lavage fluid. ${ }^{32}$

A safety evaluation of aviptadil was performed by conducting five Phase II trials under the observation the European Medicines Agency (EMA) and aviptadil was found to be a well-tolerated drug, with fewer side effects such as hypotension, flushing, and diarrhoea. Another open label Phase I study was conducted in 2005 
with 8 patients suffering from sepsis-related ARDS (all were on mechanical ventilation), who had been treated with an intravenous aviptadil infusion over 12 hours (50-100 pmol/kg/hour). Seven among those eight critically ill patients were successfully taken off of the ventilator and discharged home. Other than that, no drug-related serious adverse events were recorded and serial estimation of the serum blood TNF-a level showed significant decrement at the end of the treatment. ${ }^{33}$

\section{ROLE PLAYED BY AVIPTADIL IN COVID-19}

SARS-CoV-2 infection is characterised by a hyperimmune response and dysregulated productions of cytokines and chemokines, which play a pivotal role in severe lung injury and unfavourable clinical outcomes in patients with COVID-19.34-37

Several non-structural proteins ( $\mathrm{nsp}$ ) play a significant role in SARS-CoV-2 viral RNA replication process. Among them, the SARS-CoV-2 nsp16-nsp10 complex works as a 2'-O-methyltransferase. ${ }^{38}$ This complex is also necessary to evade the immune recognition process. $^{39}$ Results of in silico structural bioinformatics analyses have demonstrated the potential sites of binding specificity between aviptadil and nsp16. The interaction model also showed the process of the initial binding of aviptadil with nsp10 and nsp16, which may inhibit the 2'-O-methyltransferase activity of the SARS-CoV-2 nsp10/16 complex. ${ }^{40}$ The SARSCoV-2 virus enters the AT-II cell through the binding of its spike protein to the angiotensinconverting enzyme 2 surface receptors. ${ }^{41}$ Unlike the AT-I cells, only AT-II cells express the VPAC1 receptor that VIP binds to, thus VIP and its analogues deserve special attention as a therapeutic option to combat the hypoxemic lung injury in COVID-19.

Aviptadil is the only pulmonary therapeutic agent to have been granted 'fast track' status by the FDA and to be allowed into both Phase II and III clinical trials, as well as an expanded use protocol for those who are unable to enter the clinical trial due to excluded comorbidity.

The initial use of aviptadil via an intravenous route for the first time (after getting the authorisation of emergency use as an investigational new drug from the FDA) was reported in a case report by Jihad Georges Youssef et al. when a double lung transplant patient (additionally in a stage of antibody-mediated rejection) got infected with SARS-CoV-2 and subsequently developed severe respiratory failure and was treated with aviptadil at Houston Methodist Hospital, Texas, USA. After the third dose of aviptadil via infusion, there was a dramatic improvement in oxygen saturation and radiographic changes, which ultimately lead to the patient being discharged from the hospital and was alive until 28 days after post-discharge, as per the latest information gathered from the study. ${ }^{22}$

In another case series, 21 consecutive lab-confirmed patients with SARS-CoV-2 and multiple comorbidities showed significant improvement both from the radiological as well as a clinical point of view after being treated with intravenous aviptadil. Most were sent back home after being weaned frommechanical ventilation and decannulated from extracorporeal membrane oxygenation support, which was also associated with biochemical improvements in the form of the steady decline of inflammatory markers (e.g., IL-6 and C-reactive protein). ${ }^{42}$

Currently, nine clinical trials are on the list (two of which are in India), where aviptadil is being tried via both the inhalational and intravenous route and subsequently tested based on some outcome parameters to assess the safety and efficacy of the drug in comparison to the use of placebo, remdesivir, monoclonal antibodies, and other immunosuppressants in the treatment of COVID-19 that is complicated by severe respiratory failure. The details of those trials are summarised in Table 2. Among those nine trials, seven are in the recruitment stage, comprising the trial with maximum sample size, where one trial is completed and the data of the remaining one is available recently.

Of all the trials mentioned in Table 2, only one trial has been completed recently (in February 2021), which demonstrated promising results on the intravenous use of aviptadil in COVID-19. In this multicentre, placebo-controlled trial, 196 patients with COVID-19 respiratory failure were randomised 2:1 to receive 3 days of intravenous aviptadil or placebo. The primary endpoint was "alive and free from respiratory failure at 
Table 1: Effect of vasoactive intestinal peptides on various experimental animal models of acute lung injury.

\begin{tabular}{|l|l|l|}
\hline Animal models tested for & Aetiopathology of lung injury & References \\
\hline Rat & NDMD induced lung injury with arginine & Said (1996); Dickman et al. (2000) ${ }^{24}$ \\
\hline Guinea pig & Paraquat (methyl viologen) & Pakbaz et al. (1993)25 \\
\hline Rat & Hydrochloric acid induced pulmonary oedema & Foda (1988) ${ }^{26}$ \\
\hline Rat & Cobra venom factor model of septic shock & Mulligan (1992)27 \\
\hline
\end{tabular}

NDMD: N-dimethyl daunomycin.

Table 2: Summary of current trials of aviptadil in COVID-19.

\begin{tabular}{|c|c|c|c|c|}
\hline Trial identifier & Drugs used & Location & Recruitment status & Estimated sample size \\
\hline NCTO4844580 43 & Aviptadil; placebo & Turkey & Recruiting & 80 \\
\hline NCTO4536350 44 & Aviptadil; placebo & Switzerland & Recruiting & 82 \\
\hline NCTO436009645 & Aviptadil; placebo & USA & Recruiting & 144 \\
\hline NCTO484376146 & $\begin{array}{l}\text { Aviptadil; remdesivir; } \\
\text { placebo }\end{array}$ & USA & Recruiting & 640 \\
\hline NCTO448808147 & $\begin{array}{l}\text { Aviptadil; remdesivir; } \\
\text { pulmozyme; } \\
\text { celecoxib; famotidine; } \\
\text { narsoplimab; } \\
\text { yclosporine; IC14 }\end{array}$ & USA & Recruiting & 1,500 \\
\hline NCTO431169748 & Aviptadil; placebo & USA & Completed & 196 \\
\hline NCTO445383949 & Aviptadil & USA & Available & 196 \\
\hline CTRI/2021/O4/03311850 & Aviptadil; placebo & India & Recruiting & 150 \\
\hline CTRI/2021/06/03437351 & Aviptadil; placebo & India & Recruiting & 152 \\
\hline
\end{tabular}

day 60." The investigators also studied the mechanistic effect of aviptadil on blocking cytokine production and its link to survival and recovery from respiratory failure. It was found that, when controlling for baseline severity and site of care, patients treated with aviptadil were significantly more likely to be alive and free from respiratory failure at 60 days compared with those treated with placebo $(p=0.02)$ and demonstrated significance on numerous other clinical endpoints. Without controlling for the site of care, a two-fold increased odds of survival was seen at 60 days (95\% confidence interval: 1.0-3.9; $\mathrm{p}=0.035)$. Biomarker analysis demonstrates that aviptadil significantly decreased the probability of an IL-6 increase relative to placebo (50\% versus $71 \% ; \mathrm{p}=0.04$ ) and that preventing this cytokine rise was highly correlated with survival 
and recovery $(p<0.0001)$, regardless of baseline severity or treatment site. ${ }^{52}$

As the study is limited by its insufficient power and other important outcome analysis as well as the 60-day endpoint assessment as reports are still awaited, it is too early to conclude the study objective of this trial. It is also true that, as per the available data about the use of this molecule in ARDS-related sepsis as well as its use in the preclinical lung injury model, this molecule retains the hope to become an effective treatment option in the management of respiratory failure caused by SARS-CoV-2 infection.

\section{CONCLUSION}

Aviptadil, as a synthetic VIP, holds a promising place in the armamentarium of treatment of SARS-CoV-2. The class effect of this drug is already established in the almost similar clinical scenario of ARDS caused by sepsis as well as other related lung injuries in preclinical models. The safety data of this molecule has also had a favourable notation on the use in several respiratory airway diseases. Current interim analysis data about the safety and efficacy of this molecule is encouraging in spite of limitations regarding the sample size of the study affecting the power. Upcoming results from the ongoing clinical trials will play a pivotal role in treatment policy-making aspects. More robust data on a larger target population will be immensely helpful to prove its impact on the reduction of the disease burden in the treatment of this deadly virus.

\section{References}

1. Chacko J, Unais M. Pharmacologic treatment of COVID-19: evidencebased update. Indian J Respir Care. 2021:10(Suppl 1):34-8.

2. Heustess $A M$ et al. Clinical management of COVID-19: a review of pharmacological treatment options. Pharmaceuticals (Basel). 2021;14(6):520.

3. Said SI, Mutt V. Potent peripheral and splanchnic vasodilator peptide from normal gut. Nature. 1970;225:863-4.

4. Li L et al. Vasoactive intestinal polypeptide induces surfactant protein A expression in ATII cells through activation of $\mathrm{PKC} / \mathrm{C}-\mathrm{Fos}$ pathway. Peptides. 2010;31(11):2016-

5. Mossel EC et al. SARS-CoV replicates in primary human alveolar Type II cell cultures but not in Type I-like cells. Virology. 2008;372:127-35.

6. Tang $\mathrm{H}$ et al. Neuropeptide regulation of cytokine expression: effects of VIP and Ro 25-1553. J Interferon Cytokine Res. 1995;15(11):993-1003.

7. Voice JK et al. Roles of vasoactive intestinal peptide (VIP) in the expression of different immune phenotypes by wild-type mice and $\mathrm{T}$ cell targeted Type II VIP receptor transgenic mice. J Immunol. 2003;170(1):308-14

8. Gonzalez-Rey E, Delgado M. Vasoactive intestinal peptide and regulatory $\mathrm{T}$-cell induction: a new mechanism and therapeutic potential for immune homeostasis. Trends Mol Med. 2007;13(6):241-51.

9. Delgado $M$ et al. Vasoactive intestinal peptide (VIP) and pituitary adenylate cyclase-activation polypeptide (PACAP) protect mice from lethal endotoxemia through the inhibition of TNF- $a$ and IL-6. J Immunol. 1999;162(2):1200-5.

10. Berisha $\mathrm{HI}$ et al. New evidence for transmitter role of VIP in the airways: impaired relaxation by a catalytic antibody. Pulm Pharmacol Ther. 2002:15(2):121-7.

11. Sharma V et al. Granzyme B, a new player in activation-induced cell death is downregulated by vasoactive intestinal peptide in Th2 but not Th1 effectors. J Immunol. 2006;176(1):97110 .

12. Said SI. Vasoactive intestinal peptide in the lung. Ann N Y Acad Sci. 1988:527(1):450-64.

13. Hasaneen NA et al. Nitric oxide and vasoactive intestinal peptide as cotransmitters of airway smooth-muscle relaxation: analysis in neuronal nitric oxide synthase knockout mice. Chest. 2003;124(3):1067-72.

14. Saga T, Said SI. Vasoactive intestinal peptide relaxes isolated strips of human bronchus, pulmonary artery, and lung parenchyma. Trans Assoc Am Physicians. 1984:97:304-10.

15. Nagahiro I et al. Vasoactive intestinal peptide ameliorates reperfusion injury in rat lung transplantation. J Heart Lung Transplant. 1998;17(6);617-21

16. Virgolini I et al. Vasoactive intestinal peptide receptor scintigraphy. J Nucl Med. 1995;36(10):1732-9

17. Mathioudakis A et al. Vasoactive intestinal peptide inhaled agonists: potential role in respiratory therapeutics. Hippokratia. 2013;17(1):12-6.

18. Pakbaz H et al. VIP enhances and nitric oxide synthase inhibitor reduces survival of rat lungs perfused ex vivo. Ann N Y Acad Sci. 1994;723:426-8.

19. Li L et al. Role of $\mathrm{C}$-fos gene in vasoactive intestinal peptide promoted synthesis of pulmonary surfactant phospholipids. Regul Pept. 2007;140(3):117-24.

20. Delgado $\mathrm{M}$ et al. Vasoactive intestinal peptide and pituitary adenylate cyclase-activating polypeptide inhibit tumor necrosis factor atranscriptiona activation by regulating nuclear factor-kB and cAMP response element-binding protein/c-Jun. J Biol Chem. 1998;273(47):31427-36.

21. Mason RJ. Pathogenesis of COVID-19 from a cell biology perspective. Eur Respir J. 2021;55(4):2000607.

22. Onoue $\mathrm{S}$ et al. Vasoactive intestinal peptide and pituitary adenylate cyclase-activating polypeptide attenuate the cigarette smoke extract-induced apoptotic death of 
rat alveolar L2 cells. Eur J Biochem. 2004;271(9):1757-67.

23. Temerozo JR et al. The neuropeptides VIP and PACAP inhibit SARS-CoV-2 replication in monocytes and lung epithelial cells and decrease the production of proinflammatory cytokines in infected cells. 2020;DOI:1 0.1101/2020.07.25.220806.

24. Dickman KG et al. Ionotropic glutamate receptors in lungs and airways: molecular basis for glutamate toxicity. Am J Respir Cell Mol Biol. 2004;30(2):139-44.

25. Pakbaz $\mathrm{H}$ et al. Paraquat-induced lung injury: prevention by vasoactive intestinal peptide and related peptide helodermin. Am J Physiol. 1993;265(4 Pt 1):L369-73.

26. Foda HD et al. Vasoactive intestinal peptide protects against $\mathrm{HCl}$-induced pulmonary edema in rats. Ann N Y Acad Sci. 1988;527(1):633-6.

27. Mulligan MS et al. Role of leukocyte adhesion molecules in complementinduced lung injury. J Immunol. 1993;150(6):2401-6.

28. Li L et al. Effect of vasoactive intestinal peptide on pulmonary surfactants phospholipid synthesis in lung explants. Acta Pharmacol Sin. 2004;25(12):1652-8.

29. Hashimoto $S$ et al. Upregulation of two death pathways of perforin/ granzyme and FasL/Fas in septic acute respiratory distress syndrome. Am J Respir Crit Care Med. 2000;161(1):237-43.

30. Javitt JC. Perspective: the potential role of vasoactive intestinal peptide in treating COVID-19. Authorea. 2020:DOI:10.22541/ au.158940764.42332418.

31. Raveendran AV et al. Role of Aviptadil in COVID-19. BMH Med J. 2021;8(2):77-83.

32. Leuchte $\mathrm{HH}$ et al. Inhalation of vasoactive intestinal peptide in pulmonary hypertension. Eur Respir J. 2008;32(5):1289-94.

33. Prasse $A$ et al. Inhaled vasoactive intestinal peptide exerts immunoregulatory effects in sarcoidosis. Am J Respir Crit Care
Med. 2010;182(4):540-8.

34. Youssef JG. Treatment of sepsisrelated acute respiratory distress syndrome with vasoactive intestinal peptide. 2020. Available at: https:// papers.ssrn.com/sol3/papers. cfm?abstract id=3662952. Last accessed: 13 December 2021.

35. Chen $\mathrm{G}$ et al. Clinical and immunological features of severe and moderate coronavirus disease 2019. J Clin Invest. 2020;130(5):2620-9.

36. Tang $D$ et al. The hallmarks of COVID-19 disease. PLoS Pathog. 2020;16(5):e1008536.

37. Ragab D et al. The COVID-19 cytokine storm; what we know so far. Front Immunol. 2020;11:1446.

38. Giamarellos-Bourboulis EJ et al. Complex immune dysregulation in COVID-19 patients with severe respiratory failure. Cell Host Microbe. 2021;27(6):992-1000.e3.

39. Bouvet $\mathrm{M}$ et al. In vitro reconstitution of SARS-coronavirus mRNA cap methylation. PLoS Pathog. 2010;6(4):e1000863.

40. Bollati Met al. Recognition of RNA cap in the Wesselsbron virus NS5 methyltransferase domain: implications for RNA-capping mechanisms in flavivirus. $\mathrm{J}$ Mol Biol. 2009;385(1):140-52.

41. Alnomasy SF et al. Inhibitory effects of aviptadil on the SARS-COV-2 nsp10/ nsp16 protein complex. 2021;DOI:10.21203/rs.3.rs-191980/v1.

42. Youssef JG et al. Brief report: rapid clinical recovery from critical COVID-19 with respiratory failure in a lung transplant patient treated with intravenous vasoactive intestinal peptide. 2020;DOI:10.20944/ preprints202007.0178.v1.

43. Centurion Pharma. A clinical study evaluating inhaled aviptadil on COVID-19 (HOPE). NCTO4844580. https://www.clinicaltrials.gov/ct2/ show/NCTO4844580.

44. Jörg Leuppi. Inhaled aviptadil for the treatment of COVID-19 in patients at high risk of ARDS. NCTO4536350. https://clinicaltrials.gov/ct2/show/ NCTO4536350.
45. NeuroRx, Inc. Inhaled ZYESAMI'M (aviptadil acetate) for the treatment of severe COVID-19 (AVICOVID-2). NCTO4360096. https://clinicaltrials. gov/ct2/show/NCTO4360096.

46. National Institute of Allergy and Infectious Diseases (NIAID). ACTIV3b: therapeutics for severely ill inpatients with COVID-19 (TESICO). NCT04843761. https://clinicaltrials. gov/ct2/show/NCT04843761.

47. QuantumLeap Healthcare Collaborative. I-SPY COVID-19 TRIAL: an adaptive platform trial for critically ill patients (I-SPY_COVID). NCT04488081. https://clinicaltrials. gov/ct2/show/NCT04488081.

48. NeuroRx, Inc. Intravenous aviptadil for critical COVID-19 with respiratory failure (COVID-AIV). NCTO4311697. https://www.clinicaltrials.gov/ct2/ show/NCT04311697.

49. NeuroRx, Inc. ZYESAMI (Aviptadil) intermediate population expanded access protocol (SAMICARE). NCTO4453839. https://clinicaltrials. gov/ct2/show/NCTO4453839.

50. Zuventus Healthcare Limited. A clinical trial of intravenous aviptadil along with standard treatment in severe COVID-19 patients with respiratory failure. CTRI/2021/04/033118. http://www. ctri.nic.in/Clinicaltrials/pmaindet2. php?trialid=53211.

51. Mandya Institute of Medical Sciences. A clinical trial of intravenous aviptadi along with standard treatment in subjects hospitalized with respiratory failure/acute respiratory distress syndrome associated with severe coronavirus disease (COVID-19). CTRI/2021/06/034373. http://www. ctri.nic.in/Clinicaltrials/pmaindet2. php?trialid $=57078$

52. Youssef JG et al. Intravenous aviptadil is associated with increased recovery and survival in patients with COVID-19 respiratory failure: results of a 60-day randomized controlled trial. Available at: https://papers. ssrn.com/sol3/papers.cfm?abstract id=3830051. Last accessed: 13 December 2021. 